The Psychological Record, 2008, 58, 443-464

\title{
A COMPARISON OF "DIRECT” VERSUS “DERIVED” EXTINCTION OF AVOIDANCE RESPONDING
}

\author{
Bryan. T. Roche \\ National University of Ireland, Maynooth \\ Jonathan W. Kanter and Keri R. Brown \\ University of Wisconsin-Milwaukee \\ Simon Dymond \\ University of Wales, Swansea \\ Ciara C. Fogarty \\ National University of Ireland, Maynooth
}

\begin{abstract}
To establish a series of derived relations between arbitrary stimuli, 20 subjects were exposed to nonarbitrary and arbitrary relational training and testing procedures. Subjects were then exposed to an avoidance conditioning procedure in which one member from each relation was established as a discriminative stimulus for avoidance and nonavoidance, respectively. Subjects who showed conditioned avoidance and nonavoidance also showed derived avoidance and nonavoidance to other relation members. All subjects were then exposed to one of two extinction procedures, in which the original discriminative stimuli or other class members were employed, respectively. The majority of subjects exposed to both procedures also demonstrated spontaneous derived extinction of avoidance responding. These findings may have several important treatment implications.
\end{abstract}

Several decades of research on stimulus equivalence have demonstrated that verbally able humans can relate stimuli in the absence of direct training after being taught a series of related conditional discriminations. For example, if a subject is taught to relate A to B and A to C, the subject will likely relate $\mathrm{B}$ to $\mathrm{A}, \mathrm{C}$ to $\mathrm{A}, \mathrm{B}$ to $\mathrm{C}$, and $\mathrm{C}$ to $\mathrm{B}$ without any additional training. One interesting property of these derived relations is that often a response trained to one member of an equivalence class will transfer to other members of that class without any direct training and even though the class members share no formal properties (e.g., Dougher \& Markham, 1994). Several studies

Correspondence concerning this article should be addressed to Bryan Roche, Department of Psychology, National University of Ireland, Maynooth, Co. Kildare, Ireland. E-mail: Bryan.T.Roche@nuim.ie 
have demonstrated such a transfer of function after equivalence training, including demonstrations of transfer of discriminative functions (e.g., Barnes \& Keenan, 1993; de Rose, McIlvane, Dube, Galpin, \& Stoddard, 1988; Lazar, 1977); ordinal functions (Green, Sigurdardottir, \& Saunders, 1991); conditional stimulus control (Wulfert \& Hayes, 1988); contextual control (Gatch \& Osborne, 1989; Kohlenberg, Hayes, \& Hayes, 1991); and conditioned reinforcement and punishment functions (Hayes, Kohlenberg, \& Hayes, 1991).

Of particular clinical interest may be the transfer of aversive elicitation, avoidance, and extinction functions, which are seen as fundamental to the nature and treatment of anxiety disorders. Specifically, the traditional behavioral model of anxiety held that anxiety results from classical conditioning in which previously neutral stimuli come to elicit arousal after being paired with unconditioned aversive stimuli. Subsequently, these nowconditioned aversive stimuli evoke avoidance, which is maintained by negative reinforcement (Mowrer, 1960; Wolpe, 1958). For example, a previously neutral snake becomes a conditioned aversive stimulus after one is bitten and hurt by that snake. This snake, and possibly others, now evokes avoidance, which is maintained because anxious arousal elicited by snakes can be reduced or avoided altogether if one successfully avoids snakes. Exposure treatments, in turn, were hypothesized to work by blocking avoidance and repeatedly presenting the previously neutral stimuli until extinction of the aversive response occurred (Barlow, 2002). This theory was criticized on many fronts, including the obvious clinical evidence that many anxious patients do not have the direct conditioning histories posited as necessary by the theory (many people are scared of snakes even though they have never been bitten), and that extinction does not reliably occur upon natural exposure to the conditioned stimulus alone (many snake-phobic people are repeatedly exposed to snakes but remain snake phobic: Rachman, 1977; 1991).

The key issue here is whether elicitation, avoidance, and extinction responses can transfer across members of equivalence classes without direct training. Demonstrations of such transfers of function would suggest that to the degree that research on stimulus equivalence and related research on Relational Frame Theory (RFT: Hayes, Barnes-Holmes, \& Roche, 2001) present a valid analog of human language and cognition, language processes may be responsible for the occurrence of anxiety-related functional responses in the absence of direct conditioning histories. This would add considerably to the traditional behavioral account of anxiety and would potentially offer a behavioral counterpart to the cognitive theories that arose in part as a response to criticisms of the original behavioral model (Forsyth, 2000; Forsyth \& Eifert, 1996; Friman, Hayes, \& Wilson, 1998). Such research may have broader clinical implications, since avoidance is increasingly seen as important to depressive disorders as well (Barlow, Allen, \& Choate, 2004; Ottenbreit \& Dobson, 2004). In fact, Hayes, Wilson, Gifford, Follette, and Strosahl (1996) have argued that avoidance of aversive, presumably elicited, private events may be a broad functional process key to many topographically-defined diagnostic categories.

Dougher, Augustson, Markham, Greenway, and Wulfert (1994) first demonstrated the transfer of eliciting functions through equivalence classes. Using match-to-sample procedures, 8 subjects were taught two 4-member equivalence classes. After learning these classes, 1 member of one class was paired with electric shock. Subjects were then exposed to the other members to test for transfer of elicited arousal, measured via skin conductance. Of 
the 8 subjects, 6 displayed elevated skin conductance responses to other members of the class that had not been directly paired with the shock, thus demonstrating transfer of eliciting functions in the majority of subjects.

A second experiment demonstrated that extinction of elicitation functions can also transfer through equivalence classes (Dougher et al., 1994). In this experiment, an additional 8 subjects were taught two 4-member equivalence classes, and then all 4 members of one class were paired with electric shock. Next, 1 member of that class was presented in extinction; then subjects were exposed to the other class members to test for transfer of extinction functions, measured by the failure to elicit skin conductance. All 8 subjects demonstrated such transfer.

Augustson and Dougher (1997) subsequently demonstrated that avoidance responding may transfer through equivalence classes as well. As in the earlier experiments (Dougher et al., 1994), 8 subjects were trained in two 4-member equivalence classes using a match-to-sample procedure. Then, electric shock was paired with 1 member of one class, and subjects were taught that they could avoid this member by repeatedly pressing a key on the keyboard. Subsequently, subjects were exposed to the other class members to test for a transfer of avoidance functions. Transfer of avoidance response functions was demonstrated in all 8 subjects.

Although these previous studies investigated transfer of function within the framework of equivalence classes, in RFT equivalence is viewed as just one type of relational frame (i.e., sameness); and deriving relations among stimuli in the absence of direct conditioning is viewed as a generalized operant (Barnes-Holmes \& Barnes-Holmes, 2000). Responding in accordance with other derived relations has also been demonstrated, including relations of sameness, opposition, and difference (Steele \& Hayes, 1991; Roche \& Barnes, 1996, 1997; Whelan \& Barnes-Holmes, 2004); more than and less than (Dymond \& Barnes, 1995; O' Hora, Roche, Barnes-Holmes, \& Smeets, 2002; Whelan, Barnes-Holmes, \& Dymond, 2006); and before and after (O'Hora, Barnes-Holmes, Roche, \& Smeets, 2004; see also Barnes \& Roche, 1996; Hayes \& Barnes, 1997). Evidence is mounting that these relations may result in the transformation of functions in accordance with the relations trained, akin to the transfer of function seen with equivalence relations (for a review, see Dymond \& Rehfeldt, 2000).

Recently, Dymond, Roche, Forsyth, Whelan, and Rhoden (2007) replicated and extended Augustson and Dougher's (1997) study using the relational frames of sameness and opposition. They first trained subjects using the relational pretraining developed by Steele \& Hayes (1991) to establish contextual (relational) control of Same and Opposite for two arbitrary stimuli. They then exposed subjects to a series of contextually- controlled conditional discriminations using these contextual cues. This involved training subjects to choose $\mathrm{B} 1$ and $\mathrm{Cl}$ given A1 in the presence of the Same cue, and to choose B2 and C2 given A1 in the presence of the Opposite cue. In simple terms, the researchers trained subjects to respond to $\mathrm{B} 1$ and $\mathrm{C} 1$ as the same as $\mathrm{A} 1$ and to respond to $\mathrm{B} 2$ and $\mathrm{C} 2$ as the opposite of A1. (Nonsense syllables were used in the actual experiment, but are referred to here by alphanumeric labels.) The authors hypothesized that if B1 was then established as a discriminative stimulus for avoidance, $\mathrm{Cl}$ should also evoke avoidance (i.e., $\mathrm{B} 1$ and $\mathrm{Cl}$ are the same as each other because they are both the same as A1), whereas C2 should not (i.e., B1 and C2 are opposite to each other, because $\mathrm{Cl}$ is the same as $\mathrm{A} 1$, and $\mathrm{C} 2$ is the opposite of $\mathrm{A} 1$ ). 
The avoidance conditioning procedures of Dymond et al. (2007) differed somewhat from those used by Augustson and Dougher (1997). Specifically, during the conditioning phase, subjects were presented with either B1 or B2 in a quasi-random order for 3 s and with an inter-trial interval of 10-30s. A photograph of a bodily mutilation followed B1 $75 \%$ of the time and a blank screen 25\%. An aesthetically appealing photograph followed B2 75\% of the time and a blank screen 25\%. Subjects were instructed that they could avoid the presentation of a photograph before it was displayed by pressing the space bar while the stimulus (i.e., B1 or B2) was present on the screen. To probe for derived avoidance responding, subjects then were presented with either $\mathrm{C} 1$ or $\mathrm{C} 2$ (which had never been paired with photographs) and given the same instructions to press the space bar if they wished to avoid viewing photographs. All but one of the subjects who showed conditioned avoidance to B1 also showed differential derived avoidance to C1 but not to C2. Subjects who were exposed only to the avoidance conditioning and derived transformation phases did not demonstrate the predicted transformation.

Dymond et al. (2007) were the first to demonstrate the derived transformation of avoidance functions in accordance with a complex relational network, thus extending the behavior-analytic analog of derived fear and avoidance established for simpler equivalence relations by Dougher et al. (1994) and Augustson and Dougher (1997). For some individuals, this expanded model may more closely approximate the clinical situation. Consider an individual who has become phobic of snakes after being bitten by a friend's pet snake and now avoids the alley behind his house in fear of a snake even though he has never seen a snake in that alley or any alley and has never had a bad experience in that or a similar alley. A simple stimulus generalization account for this avoidance is difficult to construct because the alley is not formally similar to the environment where the snake bite occurred. Instead, the individual may have derived verbally that snakes may be found in alleys. This derived relations approach to avoidance suggests that a myriad of formally dissimilar events can be established as discriminative stimuli for avoidance by virtue of a single avoidance response established for a single member of a complex network of verbal stimuli.

However, a complete model of avoidance must do more than simply provide a conceptually coherent account of the etiology of avoidance disorders. It should also analog treatment processes based on that account. In particular, traditional behavioral treatments for anxiety disorders rest on the assumption that a history of direct conditioning is responsible for the acquisition of the aversive elicited response and subsequent avoidance. Therefore, repeated, invivo, nonreinforced exposure to the conditioned stimulus to achieve extinction of the anxiety-related response functions became the preferred behavioral treatment for several anxiety disorders (Barlow, 2002). However, to the extent that verbal processes are at work and the targeted response functions are derived as well as directly conditioned, extinction of directly-conditioned responses may not be necessary. In these cases, extinction of derived responding may be as effective as extinction of directly-conditioned responding.

The intent of the current study was to replicate and extend the studies of Augustson and Dougher (1997) and Dymond et al. (2007) by demonstrating derived avoidance in accordance with derived relations other than equivalence, and to examine the relative effectiveness of two different interventions for derived avoidance in a laboratory context. 


\section{Method}

\section{Subjects}

For this experiment, 20 subjects (10 female, 10 male) over the age of 18 were recruited through personal contacts. Before the experiment, all subjects provided informed consent to view a series of photographs, including images of bodily mutilations. They were explicitly told that they could discontinue participation at any time during the experiment, although none chose to do so. The experiment required 1-2 hr to complete, depending on the subject's performance during the relational training and testing phases. Upon completion of the study, all subjects were fully debriefed.

\section{Setting, Apparatus, and Stimuli}

Sessions were conducted in a small room $(4 \times 3$ meters). The subject was seated at a desk holding a computer monitor and a keyboard that displayed black characters on a white background. PsyScope (version 1.2.2: Cohen, MacWhinney, Flatt \& Provost, 1993) controlled all stimulus presentations and recordings of responses.

Two stimuli, each consisting of six characters, !!!!! and ??????, were used as contextual cues. For each subject, these stimuli were randomly assigned to the roles of same and opposite. Seven sets of arbitrarily configured visual figures served as stimuli during relational pretraining and testing (see next section). Seven nonsense syllables (i.e., CUG, JOM, PAF, MEL, ZID, LEB, \& VEP) were employed for relational training and testing. These were randomly assigned as sample and comparison stimuli and are labeled, for clarity, using these alphanumerics: A1, B1, B2, C1, C2, N1, and N2, respectively. Finally, aversive and aesthetically pleasing pictures used for avoidance conditioning were taken from the International Affective Picture System (IAPS: Lang, Bradley, \& Cuthbert, 2001). This is a collection of normative emotional stimuli that are widely used in psychological research. A total of 20 pictures was selected, 6 pleasant photos (e.g., balloons) and 6 aversive photos (e.g., severely injured/mutilated human bodies and faces).

\section{Procedure}

General experimental sequence. Each subject signed a consent form, acknowledging the distasteful nature of the aversive pictures to be used during the avoidance conditioning phase, at least $24 \mathrm{hr}$ before the experiment. This allowed the subject time to withdraw from the experiment in the interim if he or she wished. All subjects were exposed individually to the experimental procedures, and times were arranged so that subjects did not meet each other in the vicinity of the laboratory. Upon arrival to the laboratory, the subject was then seated comfortably in front of the computer screen. Each subject was then exposed to six phases: (1) nonarbitrary relational pretraining and testing, (2) arbitrary relational training and testing, (3) avoidance conditioning, (4) probe for derived avoidance responding, (5) extinction of avoidance responding, and (6) postextinction probe for avoidance responding (see Figure 1). Instructions always began each phase of the experiment. 
Phase 1: Nonarbitrary Relational Training and Testing

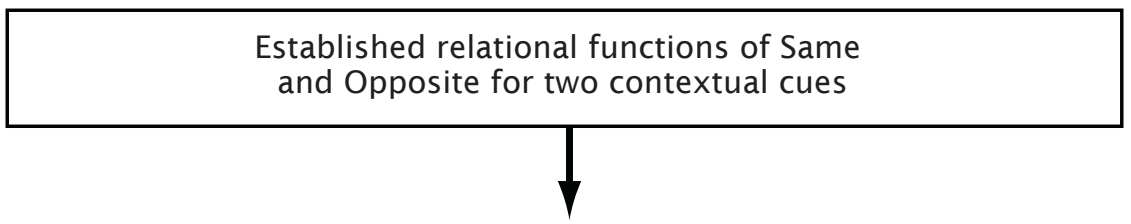

Phase 2: Arbitrary Relational Training and Testing

Established relational network that led to the emergence of the derived relations:

B1 Same as C1

B2 same as C2

Phase 3: Avoidance Conditioning

Established avoidance functions for B1

Established non-avoidance functions for B2

Phase 4: Probes for Derived Avoidance

Tested for derived avoidance of $\mathrm{C} 1$

Tested for derived non-avoidance of C2

Phase 5: Extinction of Avoidance

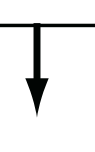

B1 and B2 presented in Extinction (Direct Extinction)

or

$\mathrm{C} 1$ and $\mathrm{C} 2$ presented in Extinction (Derived Extinction)

Phase 6: Post-Extinction Probes for Avoidance

B1, B2, C1 and C2 presented to probe

for extinction and derived extinction of avoidance.

Figure 1. A schematic outline of the general experimental sequence. 
Phase 1: Nonarbitrary relational training and testing. During Phase 1 , subjects were presented with a series of match-to-sample tasks on the computer screen. They were first given the following instructions:

In a moment, some images will appear on the screen. Your task is to look at the image at the top of the screen, then look at the image in the middle of the screen, and, finally, look at the three images at the bottom of the screen. You should choose one of the three images by pressing the Z, V, or M key on the keyboard in front of you. If you want to choose the image on the left, press the $\mathrm{Z}$ key on the left. If you want to choose the image in the middle, press the V key in the middle; and if you want to choose the image on the right, press the M key on the right. If you have any questions, ask the experimenter now.

The purpose of this phase was to establish the functions of same and opposite for the contextual cues (!!!!!! and ??????) that would be used in the subsequent phases of the experiment. The sample and comparison stimuli were related to each other along some physical dimension (see Figure 2). Subjects were first shown a contextual cue (!!!!!! or ??????) that appeared in the center, top third of the computer screen. After 1s, a sample stimulus (e.g., a short line) appeared in the middle of the screen. Next, 1s later, three comparison stimuli (e.g., one short, one medium, and one long line) appeared in a row at the bottom of the screen. Subjects then had to choose one of the comparison stimuli. For example, in the presence of the same contextual cue, given a shortline sample stimulus, choosing the short-line comparison stimulus was reinforced. However, given the opposite contextual cue and a short line, choosing the long-line comparison stimulus was reinforced.
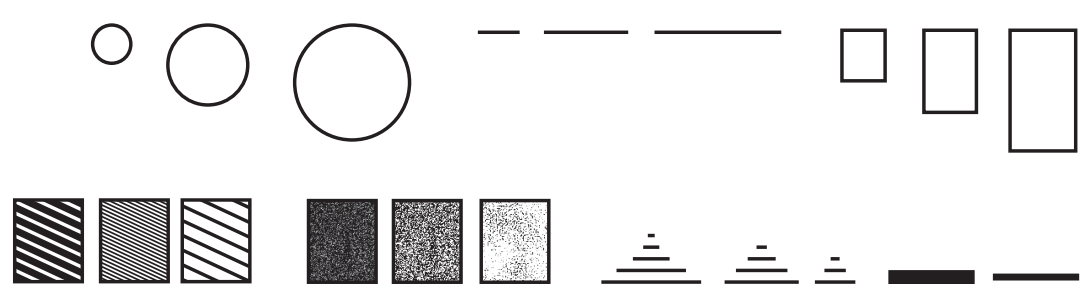

Figure 2. Nonarbitrary stimuli sets used during relational pretraining. The upper sets were employed during training. The lower sets were employed during testing.

All stimuli remained on the screen until the subject made a response by pressing $\mathrm{Z}, \mathrm{V}$, or $\mathrm{M}$ on the computer keyboard. Feedback in the form of the printed words "CORRECT" or "WRONG" was displayed for $2 \mathrm{~s}$ following responses on all training trials. The positions of correct comparison stimuli (i.e., left, middle, right) on the screen were randomized across trials.

Phase 1 training consisted initially of a sequence of 32 trials ( 8 blocks of 4 trials). Subjects were presented with 4 trials (1 block) involving circles of varying sizes (problem set 1), followed by 4 trials 
involving lines of varying lengths (problem set 2). These 2 blocks of 4 trials were presented repeatedly in a quasi-random order until subjects had been exposed to 32 trials in total. To proceed from the pretraining to the testing phase, subjects were required to produce 16 consecutive correct responses on the final 16 trials. If subjects failed to do so, they repeated this pretraining sequence up to a maximum of four times. Subjects who were not able to pass the pretraining phase after four sequences were thanked for their time, and their participation in the study was ended.

After passing Phase 1 training, the subject was presented with further instructions, identical to those delivered before training. This represented the beginning of the testing phase. Testing consisted of a sequence of 8 trials ( 2 blocks of 4 trials) of novel problem sets (e.g., 4 trials of squares with various shading followed by 4 trials of triangles of varying sizes) with no feedback provided after responding. To proceed to Phase 2, subjects were required to produce at least 6 out of 8 correct responses. Subjects who failed to do so were retrained with a new training sequence that included one earlier problem set (e.g., problem set 2) and a completely novel set (e.g., rectangles of varying sizes). They were then re-exposed to the Phase 1 testing. Subjects could repeat this retraining sequence up to three times. After three failed testing attempts, subjects were thanked for their time and their participation in the study was ended.

Phase 2: Arbitrary relational training and testing. The purpose of this phase was to train and test for arbitrary relations involving arbitrarily related stimuli. Whereas in Phase 1 the sample and comparison stimuli were related along a physical dimension, in Phase 2 they were arbitrarily related using the same and opposite contextual cues. The probes for arbitrary relational training and testing are described according to the following convention: The contextual cue is given first in capital letters, followed by the sample stimulus, followed by the three comparison stimuli in brackets. The experimenterdesignated correct comparison is in italics. Subjects were exposed to the following four training tasks: SAME/A1 [B1-B2-N1], SAME/A1 [C1C2-N2], OPPOSITE/A1 [B1-B2-N1], OPPOSITE/A1 [C1-C2-N2]. The N1 and N2 stimuli were presented during training only and served as control stimuli to ensure that the Opposite contextual cue was functioning appropriately. More specifically, if this cue exerted mere S- control over choice of comparisons, then choosing either of two stimuli during any Opposite task would constitute a correct response. In effect, the presence of the $\mathrm{N}$ stimuli helped to establish the appropriate relational network during the training phase.

Figure 3 shows the training protocol designed to establish the relational network. Phase 2 training consisted of each of the four training tasks being presented once, each in a quasi-random order per block of 4 trials. Then 10 blocks of 4 trials were delivered across the training phase (i.e., 40 trials in total). To proceed to Phase 2 testing, subjects were required to respond correctly on all of the final 20 trials. If they failed to do so, they repeated the Phase 2 training up to a maximum of four times. Subjects who did not pass the training phase after four exposures were discontinued from the study. 


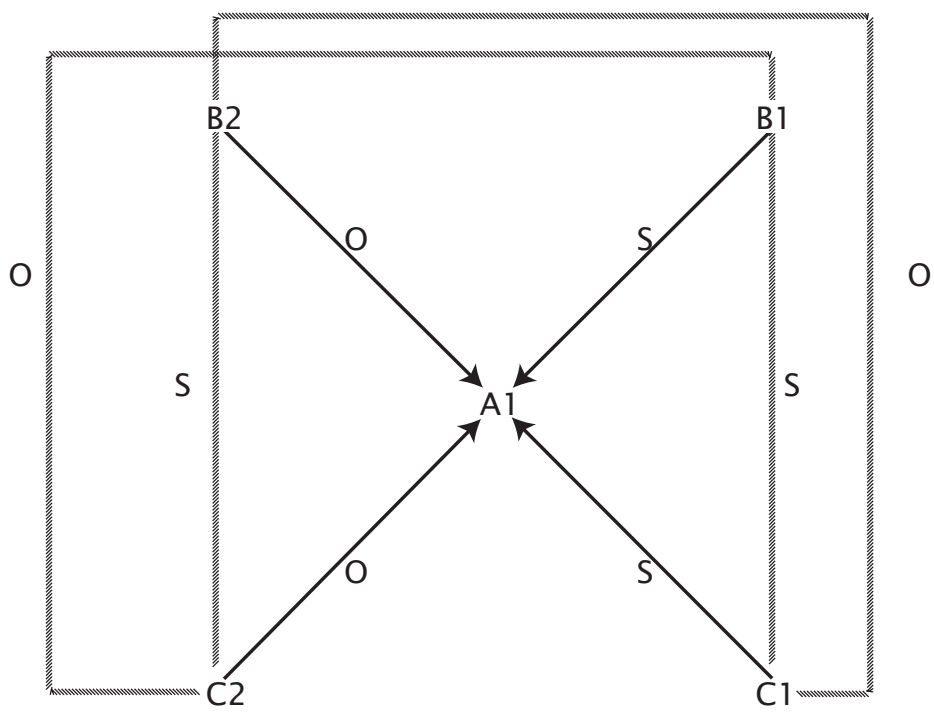

Figure 3. The network of trained and tested arbitrary relations. Solid lines indicate trained relations. Hashed lines represent derived relations.

The Phase 2 relational testing phase determined whether derived relational responding in accordance with the relations of same and opposite would emerge. Subjects were tested to determine whether they had derived the following relations: $\mathrm{B} 1$ is the same as $\mathrm{C} 1$ (not $\mathrm{C} 2$ ); $\mathrm{B} 2$ is the same as $\mathrm{C} 2$ (not C1), B1 is the opposite of C2 (not C1), and B2 is the opposite of C1 (not C2). Testing consisted of a sequence of another 40 trials administered using precisely the same format as that employed during training. To proceed to Phase 3, subjects were required to produce 38 correct responses out of 40 . Subjects who failed to respond correctly on at least 38 of the 40 testing trials repeated the relational training and were then re-exposed to the Phase 2 testing. Subjects could repeat this retraining sequence up to three times (i.e., four exposures in total) before being discontinued from the study.

Phase 3: Avoidance Conditioning. In Phase 3, avoidance functions were established for the B1 stimulus by consequating its presentation with aversive visual stimuli. These visual stimuli could be avoided by a simple operant task (i.e., pressing the space bar). Thus, B1 was established as a discriminative stimulus for avoidance. Appetitive, nonavoidance functions were established for B2. Aversive and pleasant pictures followed B1 and B2, respectively, on 75\% of trials. This was done to increase resistance to extinction so that a test for the transformation of function could be administered in which the $\mathrm{C} 1$ and $\mathrm{C} 2$ stimuli would be presented in extinction.

Before this phase began, subjects were given the following instructions:

In a moment, some images will appear on the screen. These will consist of words and pictures. Please concentrate on the screen at all times. It is important that you continue to pay attention. You may cancel a picture before it is displayed by pressing the space bar. Please be aware that some of the following pictures may be upsetting to some people. This task will continue until 
you have viewed 20 pictures. If you have any questions, please ask the experimenter now. Please press any key to continue.

Following these instructions, subjects were presented with either the $\mathrm{B} 1$ or $\mathrm{B} 2$ stimulus for $5 \mathrm{~s}$ followed by the relevant visual stimulus. These conditioning trials were presented in a quasi-random order (i.e., no more than two successive exposures to any one trial-type). Subjects could avoid the presentation of any photograph by pressing the space bar while the B1 or B2 stimulus was on the screen. If a subject pressed the space bar during this time, the screen was cleared and the words "Photograph Cancelled" appeared. If subjects did not press the space bar, either an aversive or a pleasant photograph was presented for 2s. An aversive photograph followed B1 75\% of the time, and a blank screen $25 \%$, whereas a pleasant photograph followed B2 $75 \%$ of the time and a blank screen $25 \%$. The next nonsense syllable was presented following a variable inter-trial interval of 10-30s. From now on, to aid reference to the various stimuli involved, B1 is the direct aversive stimulus and $\mathrm{B} 2$ is the direct pleasant stimulus.

Phase 3 continued until subjects viewed 20 photographs of their choosing. Thus, the number of conditioning trials varied across subjects. If subjects avoided at least 8 of the final 12 exposures to B1 and avoided no more than 2 of the final 12 exposures to B2, they proceeded to Phase 4 . Subjects were exposed to Phase 3 only once. Subjects who failed Phase 3 were discontinued from the study.

Phase 4: Probe for derived avoidance. The purpose of this phase was to test whether subjects would avoid C1 based on its relationship to B1 in the trained and testing relational network (i.e., sameness; see Figure 3). The instructions delivered were identical to those delivered prior to Phase 3. Here, C1 and C2 were presented six times each in the same manner as B1 and B2 during Phase 3. If subjects pressed the space bar during the presentation of $\mathrm{Cl}$ or $\mathrm{C} 2$, the screen was cleared and the words "Photograph Cancelled" appeared. If subjects did not press the spacebar, they were always presented with the inter-trial interval, during which the screen was blank; photographs were never presented during this phase.

If subjects produced an avoidance response on at least 4 of the 6 exposures to $\mathrm{C} 1$ and avoided no more than 2 of the 6 exposures to $\mathrm{C} 2$, they were deemed to have demonstrated derived avoidance and they proceeded to Phase 5 . Subjects were exposed to Phase 4 only once. Subjects who failed Phase 4 were discontinued from the study.

Phase 5: Extinction of avoidance. The purpose of this phase was to extinguish a subject's avoidance responding to B1 (direct extinction group) or C1 (derived extinction group). Subjects were randomly assigned to one of these two groups. Subjects in the direct extinction group were exposed to B1 and B2 for 16 trials in extinction (8 trials of each). Subjects in the derived extinction group were exposed to $\mathrm{C} 1$ and $\mathrm{C} 2$ for 16 trials in extinction ( 8 trials of each). The stimulus presentation format was identical to that employed in Phase 4. However, subjects were not provided with instructions about the avoidance of photographs, and the space bar on the computer keyboard was disabled. All stimulus presentations were followed by the normal 10-30s inter-trial interval when the screen was blank.

Phase 6: Postextinction probe for avoidance responding. The final critical stage of the experiment involved testing for avoidance to other members in the relational network (i.e., to assess whether or not B1, B2, C1, and C2 produced 
avoidance responses following the two different extinction methods). This stage evaluated (a) avoidance of the derived aversive event, C1; (b) avoidance of the directly aversive stimulus, B1; (c) avoidance of the derived pleasant stimulus, C2; and finally (d) avoidance of the directly pleasant stimulus, B2. Each stimulus was presented six times in quasi-random order for $5 \mathrm{~s}$ following a variable inter-trial interval of 10-30s. After these presentations, the following was presented on the screen: "This is the end phase of the experiment. Please contact the experimenter now."

The criterion for avoidance was a minimum of 4 avoidance responses during the 6 derived and direct aversive stimulus presentations (i.e., B1 and $\mathrm{C} 1$ ), and a maximum of 2 avoidance responses during the 6 derived or direct pleasant stimulus presentations (i.e., B2 and C2).

\section{Results}

\section{Phase 1: Nonarbitrary Relational Training and Testing}

Table 1 presents each subject's response patterns for Phases 1 and 2. In Phase 1 , Subjects $2,3,6,9,11,12,16,18$, and 19 required only one training sequence and passed the test on their first exposure. Subject 7 required training once and passed the test after two exposures. Subjects 1, 4, 5, 14, and 17 required training twice and passed the test after two exposures. Subject 8 required training twice and passed the test after two exposures, whereas the remaining Subjects 10, 13, 15, and 20 required training three times and passed the test on their first exposure.

Table 1

Number of Training and Testing Sequences Required to Pass Phases land 2

\begin{tabular}{cccccc}
\hline & \multicolumn{3}{c}{ Phase 1 } & & \multicolumn{2}{c}{ Phase 2 } \\
\cline { 2 - 3 } \cline { 5 - 6 } Subject & Training & Testing & & Training & Testing \\
\hline 1 & 2 & 1 & 2 & 2 \\
2 & 1 & 1 & 1 & 1 \\
3 & 1 & 1 & 2 & 2 \\
4 & 2 & 1 & 3 & 3 \\
5 & 2 & 1 & 2 & 2 \\
6 & 1 & 1 & 2 & 2 \\
7 & 1 & 2 & 1 & 1 \\
8 & 2 & 2 & 1 & 1 \\
9 & 1 & 1 & 1 & 1 \\
10 & 3 & 1 & 1 & 1 \\
11 & 1 & 1 & 2 & 3 \\
12 & 1 & 1 & 2 & 3 \\
13 & 3 & 2 & 3 & 4 \\
14 & 2 & 1 & 2 & 2 \\
15 & 3 & 2 & 4 & 4 \\
16 & 1 & 1 & 1 & 2 \\
17 & 2 & 1 & 2 & 2 \\
18 & 1 & 1 & 3 & 2 \\
19 & 1 & 1 & 1 & 1 \\
20 & 3 & 1 & 1 & 2 \\
\hline
\end{tabular}




\section{Phase 2: Arbitrary Relational Training and Testing}

In Phase 2 training, no subject required more than four training sequences before successfully passing. Subjects $2,7,8,9,10,16,19$, and 20 required only one exposure to the training phase. Subjects $1,3,5,6,11,12,14$, and 17 required two exposures. Subjects 4,13 , and 18 required three exposures, and Subject 15 required four exposures.

In Phase 2 testing, Subjects 2, 7, 8, 9, 10, and 19 passed testing on their first exposure. The remaining subjects were retrained and then retested. Subjects $1,3,5,6,14,16,17,18$, and 20 passed on their second exposure. Subjects 4, 11, and 12 required three exposures. Subjects 13 and 15 required four exposures.

\section{Phase 3: Avoidance Conditioning}

Table 2 shows the number of B1 and B2 trials avoided during Phase 3. Subjects 7 and 17 failed to demonstrate avoidance to B1 and were discontinued from the study. All other subjects demonstrated avoidance to B1 (direct aversive stimulus) but not to B2 (direct pleasant stimulus).

\section{Table 2}

Number of Avoidance Responses to B1 and B2 During Phase 3 (Avoidance Conditioning)

\begin{tabular}{ccc}
\hline Subject & B1 Produced Avoidance & B2 Produced Avoidance \\
\hline 1 & $16 / 17$ & $0 / 19$ \\
2 & $11 / 15$ & $0 / 16$ \\
3 & $10 / 15$ & $0 / 15$ \\
4 & $12 / 16$ & $0 / 16$ \\
5 & $13 / 16$ & $1 / 17$ \\
6 & $8 / 14$ & $0 / 14$ \\
$7^{*}$ & $2 / 11$ & $0 / 11$ \\
8 & $15 / 19$ & $1 / 19$ \\
9 & $13 / 17$ & $1 / 17$ \\
10 & $10 / 13$ & $0 / 13$ \\
11 & $10 / 15$ & $1 / 15$ \\
12 & $8 / 10$ & $0 / 10$ \\
13 & $17 / 18$ & $1 / 19$ \\
14 & $9 / 13$ & $0 / 14$ \\
15 & $14 / 16$ & $0 / 17$ \\
16 & $8 / 11$ & $0 / 12$ \\
$17^{*}$ & $0 / 10$ & $0 / 10$ \\
18 & $12 / 16$ & $0 / 16$ \\
19 & $12 / 15$ & $0 / 16$ \\
20 & $9 / 12$ & $0 / 12$ \\
\hline
\end{tabular}

* Subjects who failed this phase.

\section{Phase 4: Probe for Derived Avoidance}

Table 3 shows the number of $\mathrm{C} 1$ and $\mathrm{C} 2$ trials avoided during Phase 4. All subjects showed evidence of derived avoidance in the probe phase (avoidance testing). Subjects 6 and 9 produced the predicted derived 
avoidance response to $\mathrm{C} 1$ (the derived aversive stimulus) on every trial. Subjects $3,4,8,10,11,14$, and 15 produced the avoidance response on 5 out of the 6 trials. Last, Subjects, 1, 2, 5, 12, 13, 16, 18, 19, and 20 produced the derived avoidance response on 4 out of the 6 trials. In comparison, all subjects avoided the derived pleasant stimulus (C2) on only two or fewer occasions.

Table 3

Number of $\mathrm{Cl}$ and C2 Trials Avoided During Phase 4 (Probes for Derived Avoidance Responding)

\begin{tabular}{ccc}
\hline Subject & C1 Produced Avoidance & C2 Produced Avoidance \\
\hline 1 & $4 / 6$ & $0 / 6$ \\
2 & $4 / 6$ & $1 / 6$ \\
3 & $5 / 6$ & $0 / 6$ \\
4 & $5 / 6$ & $2 / 6$ \\
5 & $4 / 6$ & $1 / 6$ \\
6 & $6 / 6$ & $1 / 6$ \\
8 & $5 / 6$ & $0 / 6$ \\
9 & $6 / 6$ & $0 / 6$ \\
10 & $5 / 6$ & $0 / 6$ \\
11 & $5 / 6$ & $1 / 6$ \\
12 & $4 / 6$ & $2 / 6$ \\
13 & $4 / 6$ & $0 / 6$ \\
14 & $5 / 6$ & $1 / 6$ \\
15 & $5 / 6$ & $1 / 6$ \\
16 & $4 / 6$ & $1 / 6$ \\
18 & $4 / 6$ & $0 / 6$ \\
19 & $4 / 6$ & $0 / 6$ \\
20 & $4 / 6$ & $0 / 6$ \\
\hline
\end{tabular}

\section{Phase 6: Postextinction Probe for Avoidance Responding}

Direct extinction group. Table 4 presents the results of Phase 6 , where the effects of direct and derived extinction were explored. In the direct extinction group, subjects were re-exposed to the four arbitrary stimuli B1, B2, C1 and C2 after being presented with the direct aversive stimulus (B1) repeatedly in extinction. The direct extinction procedure did not extinguish avoidance responding to the direct aversive stimulus for most subjects. Subjects 1 and 2 continued to produce avoidance responses to B1 on all 6 trials. Subjects 4, 6 and 10 produced avoidance responses to B1 on 5 out of the 6 trials. Subjects 5, 8 , and 9 produced avoidance responses to B1 on 4 out of the 6 trials. Subject 3 demonstrated some extinction, producing avoidance responses to B1 on only 2 out of the 6 trials.

As expected, responding to the direct pleasant stimulus (B2) was not affected by the direct extinction procedure. Subjects $1,5,6$, and 8 produced no avoidance responses to B2 on any of the 6 trials; and the remaining Subjects $2,3,4,9$, and 10 produced avoidance responses to B2 on 1 out of the 6 trials. In total, according to our criterion for avoidance, only 1 of 9 subjects in the direct extinction group demonstrated extinction of avoidance responding to the direct aversive stimulus. 
Table 4

Response Patterns During Phase 6 (Postextinction Probe for Avoidance Responding)

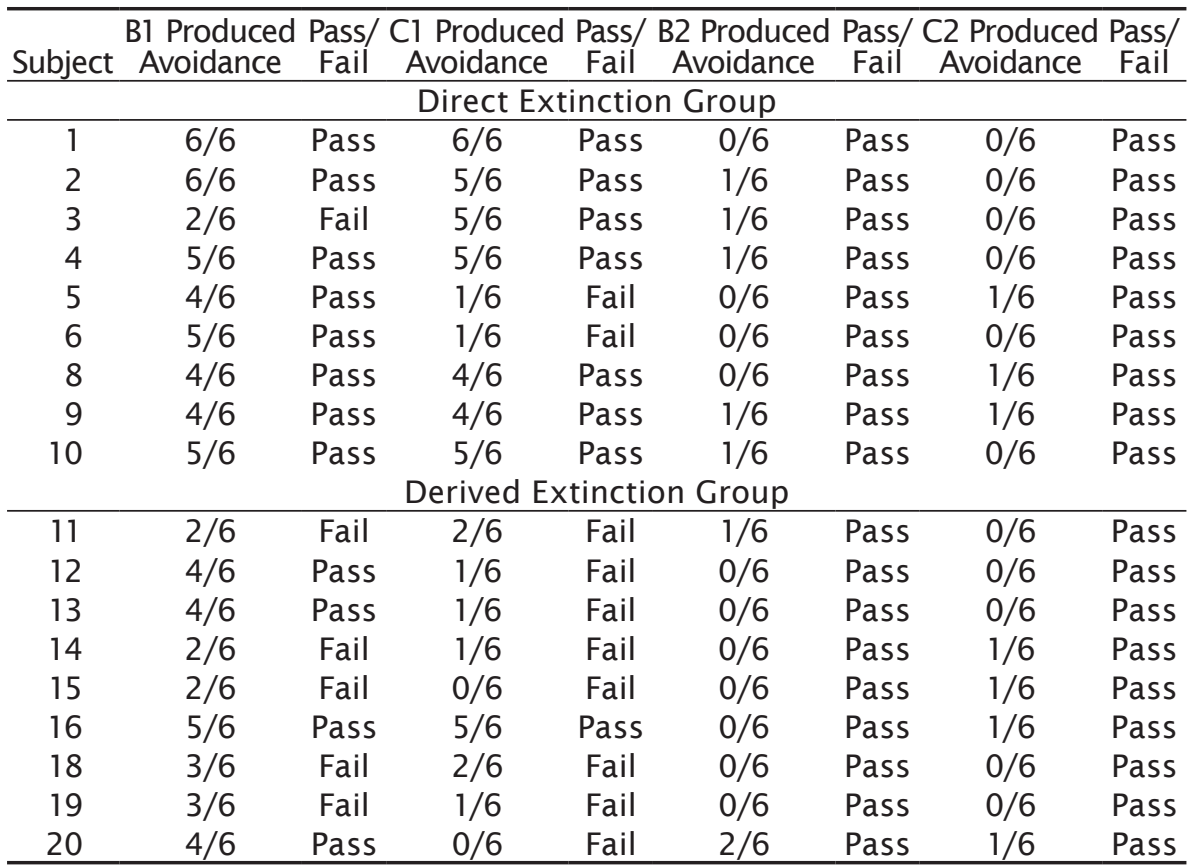

Most subjects in the direct extinction group also failed to demonstrate extinction to the derived aversive stimulus (C1) after the direct extinction procedure. Subject 1 continued to produce avoidance responses to $\mathrm{C} 1$ on all 6 trials. Subjects 2, 3, 4, and 10 produced avoidance responses to $\mathrm{C} 1$ on 5 out of the 6 trials. Subjects 8 and 9 produced avoidance responses to $\mathrm{C} 1$ on 4 out of the 6 trials. The performances of Subjects 5 and 6 did show some evidence of extinction insofar as these subjects produced avoidance responses to $\mathrm{C} 1$ on only 1 out of the 6 trials.

Responding to the derived pleasant stimulus (C2) was not affected by the direct extinction procedure. Subjects $1,2,3,4,6$, and 10 produced no avoidance responses to C2 on any of the 6 trials. Subjects 5,8 , and 9 produced avoidance responses on 1 out of the 6 trials. In total, 2 of the 9 direct extinction subjects demonstrated extinction of responding to the derived aversive stimulus.

Derived extinction group. Subjects in the derived extinction group were exposed to the derived aversive stimulus (C1) repeatedly in extinction. Of these subjects, Subject 16 continued to produce avoidance responses to the direct aversive stimulus (B1) on 5 out of the 6 trials. Subjects 12,13 , and 20 produced avoidance responses to B1 on 4 out of the 6 trials. Subjects 18 and 19 produced avoidance responses to $\mathrm{B} 1$ on 3 out of the 6 trials. The remaining Subjects, 11, 14, and 15, showed avoidance to B1 on 2 out of the 6 trials.

As expected, responding to $\mathrm{B} 2$ was not affected by the derived 
extinction procedure. Subjects $12,13,14,15,16,18$, and 19 produced no avoidance responses to B2 on any of the 6 trials. Subject 11 produced 1 avoidance response to B2 out of the 6 trials. Subject 20 produced avoidance responses to B2 on 2 out of the 6 trials. In total, 5 of the 9 derived extinction subjects demonstrated extinction of responding to the direct aversive stimulus.

The effects of the derived extinction procedure on avoidance responses to $\mathrm{C} 1$ were more marked than for $\mathrm{B} 1$. In all, 8 of the 9 subjects who received the derived extinction procedure demonstrated extinction of avoidance to C1. Specifically, Subject 16 produced avoidance responses to $\mathrm{C} 1$ on 5 out of the 6 trials. Subjects 11 and 18 produced avoidance responses to $\mathrm{C} 1$ on 2 out of the 6 trials. Subjects $12,13,14$, and 19 produced avoidance responses to $\mathrm{C} 1$ on 1 out of the 6 trials. Subjects 15 and 20 produced no avoidance responses to C1 on any of the 6 trials. Subjects 11, 12, 13, 18, and 19 produced no avoidance responses to $\mathrm{C} 2$ on any of the 6 trials. Subjects 14, 15, 16, and 20 produced avoidance responses to $C 2$ on 1 out of the 6 trials.

\section{Aggregate Results}

Four independent sample t-tests were conducted to examine these results in aggregate form. In each test, the average percent of avoidance responses of the direct extinction group was compared with those of the derived extinction group. In the first two tests, group performance was compared before the different extinction procedures were applied, in Phase 3 (for B1) and Phase 4 (for C1). In the latter two tests, avoidance of $\mathrm{B} 1$ and $\mathrm{C} 1$ was examined during the postextinction probes for avoidance responding (Phase 6). Figure 4 graphically depicts these comparisons. First, as expected, the first test showed that subjects avoided B1 the majority of the time after the aversive conditioning procedure but before extinction, and no significant differences appeared between groups (direct extinction group $M=0.76$, $S D=0.10$; derived extinction group $M=0.78, S D=0.09 ; t[16]=-0.52$, $n s)$. A pattern of responding similar to $\mathrm{C} 1$ appeared in the second test, demonstrating that derived avoidance occurred the majority of the time and no differences occurred between groups (direct extinction group $M=0.81, S D=0.13$; derived extinction group $M=0.73, S D=$ $0.08 ; t[16]=1.80, n s)$. The third test showed that the derived extinction group demonstrated more extinction of avoidance responding to B1 $(M=0.54, S D=0.18)$ than did the direct extinction group $(M=0.76$, $S D=0.21 ; t[16]=2.42, p=.028)$, thereby suggesting that the derived extinction procedure was significantly more effective in extinguishing avoidance responding to the direct aversive stimulus than was the direct extinction procedure. Finally, the derived extinction group also demonstrated more extinction of avoidance responding to $\mathrm{C} 1 \quad(M=$ $0.24, S D=0.25)$ than did the direct extinction group $(M=0.67, S D=$ $0.30 ; t[16]=3.26, p=.005)$, thus suggesting that the derived extinction procedure also was significantly more effective in extinguishing avoidance responding to the derived aversive stimulus than was the direct extinction procedure. 

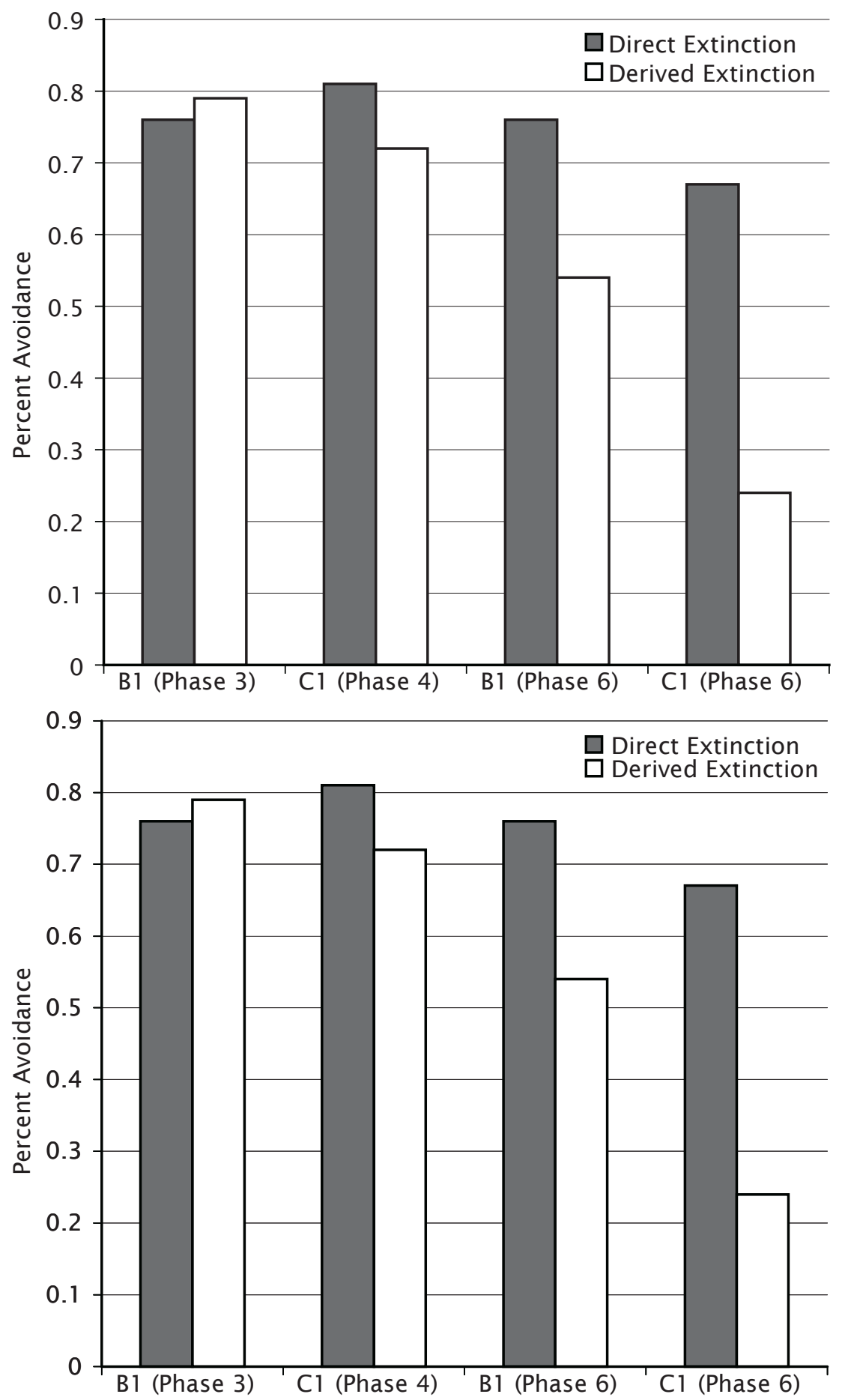

Figure 4. Percentage avoidance responses to $\mathrm{B} 1$ and $\mathrm{Cl}$ during Phases 3,4 , and 6 by group. Asterisks represent significant comparisons $(p<.05)$ 


\section{Discussion}

The present study offers two primary findings. First, the present study complements the findings of Augustson and Dougher (1997) and Dymond et al. (2007) by demonstrating the derived transfer of avoidance responses in accordance with a complex relational network. This extends the scope of a derived relations account by demonstrating that avoidance responses can emerge from even more complex derived relations between verbal stimuli than mere equivalence. Second, the present findings point us in the direction of important empirical inquiries about the effectiveness of direct and derived extinction procedures. These findings appear to suggest that extinguishing avoidance responses to stimuli that have acquired their functions indirectly is relatively easy, whereas extinction of responding to direct aversive stimuli did not occur after a fixed number (6) of extinction trials.

These findings suggest that a derived extinction procedure is more effective than a direct extinction procedure at extinguishing avoidance responses to all members of a class of discriminative stimuli for avoidance. The avoidance functions of $\mathrm{Cl}$ extinguished more readily when that stimulus was presented in extinction than did the avoidance response functions of B1 when it was presented in extinction. More specifically, subjects who received the derived extinction procedure avoided the derived aversive stimulus $24 \%$ of the time, whereas subjects who received the direct extinction procedure avoided the direct aversive stimulus $76 \%$ of the time. What is particularly interesting, however, is that the extinction of avoidance to $\mathrm{C} 1$ appears to have transferred to $\mathrm{B} 1$ for most subjects in the derived extinction group. In contrast, the minimal avoidance observed for $\mathrm{B} 1$ in the direct extinction group did not transfer to $\mathrm{C} 1$. Thus, the effect of the extinction procedure generalized across the derived relations more effectively when the derived, rather than the directly conditioned, aversive stimulus was targeted. Perhaps even more surprisingly, a greater extinction of avoidance was observed for $\mathrm{B} 1$ when $\mathrm{C} 1$ was targeted than was observed for B1 when B1 itself was targeted. More specifically, subjects in the derived avoidance group showed a derived extinction effect, whereby avoidance responding to the directly conditioned aversive stimulus extinguished spontaneously for 5 of 9 subjects. In comparison, only 2 of 9 direct extinction subjects demonstrated extinction of responding to the derived aversive stimulus. Thus, these findings suggest several ways in which derived extinction procedures may produce more powerful extinction than traditional direct extinction procedures based on widely employed exposure techniques.

It could be argued that these conclusions are compromised somewhat by the design of the study. Specifically, the derived extinction group was exposed to more extinction trials involving the $C$ stimuli than the direct extinction group. That is, for both experimental groups, Phase 4 involved the presentation of the $\mathrm{C}$ stimuli in extinction to test for the derived transformation of avoidance functions. However, continued exposure to the $\mathrm{C}$ stimuli in extinction occurred for only the derived extinction group into Phase 5. During that phase the direct extinction group was exposed to only the B stimuli in extinction. Thus, by Phase 6 the derived extinction group actually had a larger number of $\mathrm{C}$ stimulus extinction trials. This could help explain the superior extinction to the $\mathrm{C}$ stimuli observed for many of the subjects in this group. 
However, for most subjects the trials presented in the Phase 4 test for derived avoidance did not operate as extinction trials. That is, most subjects actually produced consistent avoidance responses across trials, thereby demonstrating that the avoidance responses were being negatively reinforced through the avoidance of the aversive contact with the sequelae of the C stimuli. Thus, very few trials occurred where subjects discovered that no aversive images would be presented during Phase 4 . In effect, little if any extinction occurred during Phase 4. Small differences in the number of exposures to the $\mathrm{C}$ stimuli in extinction for some subjects in the derived extinction group compared with subjects in the direct extinction group may help to explain the different effects of the extinction procedures. However, one further issue should be considered before the current effects are dismissed on the basis of slight differentials in $C$ stimulus extinction trial numbers across the experimental groups. Specifically, the foregoing issue as to differentials in C stimulus extinction trial numbers across groups also applies to the B stimuli, with the opposite implication. More specifically, during the Phase 5 extinction phase, the direct extinction group was exposed to repeated presentations of the B stimuli in extinction, whereas the derived extinction group was not. Thus, in Phase 6 the derived extinction group was exposed to the B stimuli in extinction for the first time, whereas the direct extinction group was exposed to the B stimuli in extinction for the second time. Such a differential should mean that the direct extinction group should have shown superior extinction to the B stimuli than the derived extinction group. In fact, the data showed that they did not. Indeed, they showed significantly less extinction to the B stimuli than the derived extinction group. In effect, the transfer of extinction from $C$ to $B$ for the derived extinction group was more powerful than direct extinction to B itself, despite fewer B stimulus extinction trials having been administered to the derived extinction group overall.

Although more complete extinction of avoidance was observed for the derived extinction group, complete extinction and the derived transfer of complete extinction of avoidance was not observed in the current study. This is because the current experimental preparation was designed specifically to examine relative rates of extinction using two different methods, rather than to demonstrate the transfer of extinction per se. More specifically, such a design obligated the experimenters to ensure that overall numbers of extinction trials were equal across groups. Thus, the extinction procedure could not be run to criterion to observe complete extinction for any one subject. Future research should address this issue.

This work has some clinical implications, with the caveat that replications of these findings are necessary before clinical implications can be discussed with confidence. Perhaps the most obvious clinical implication is that an avoidance response highly generalized through language processes (i.e., derived relations between words and events) may be treated best by targeting verbal stimuli in the stimulus network rather than by direct exposure to the most likely original S $S^{D}$ for avoidance. More specifically, when faced with an avoidant and anxious individual whose avoidance responses are highly generalized through language processes, targeting for extinction of the individual's fear and avoidance of objects or events related to the specific feared object or event may be more beneficial than targeting the feared object or event directly. These events may be related to the original $\mathrm{S}^{\mathrm{D}}$ for avoidance as a result of a direct history of stimulus association or via derived verbal 
relations. As an example, consider an individual who is acutely fearful and avoidant of spiders and who associates spiders verbally with hairy insects for which avoidance functions are less salient. In this case, a good starting point may be to begin extinction procedures by thinking of spiders in terms of verbal relations, rather than a class of formally related stimuli. A cursory interview can easily establish related verbal stimuli that may be targeted for extinction (e.g., centipedes) in the hope that the effects of extinction will generalize across the entire verbal relation. Targeting extinction of verbal class members rather than the original $S^{\mathrm{D}}$ for avoidance may be of particular use when the original $S^{\mathrm{D}}$ for avoidance cannot be identified by the client or therapist.

The derived extinction procedure used in this study, and suggested in the examples above, parallels a set of clinical techniques in Acceptance and Commitment Therapy (ACT: Hayes, Strosahl, \& Wilson, 1999) referred to as cognitive defusion (Masuda, Hayes, Sackett, \& Twohig, 2004). For example, one defusion technique requires the client to quickly repeat a negative selfstatement for several minutes (e.g., a socially phobic individual may repeat "I am a loser") until extinction of the statement's functions occurs. The goal of such a technique is, of course, generalization of the extinction of avoidance functions from the vocally produced stimuli to related events outside the therapy setting (e.g., feared social situations). Traditional behavior therapy might target the actual feared situation with an extinction protocol, or target a hierarchy of events formally related to the feared situation. Targeting behavior only verbally related to the feared situation is a somewhat novel move for a behavior therapist (although it is quite common for cognitive therapists). The current research provides a preliminary analog and suggests a behavioral research foundation for such a therapeutic strategy.

Direct extinction treatments are clearly effective for many problems (Barlow, 2002). The current study, however, not only provided support for the superiority of a derived extinction procedure but also failed to provide any support for a direct extinction procedure, since subjects in the direct extinction group failed to demonstrate much extinction with a simple and easily established avoidance response. The use of a 75\%-probability negative reinforcement schedule during the avoidance training stage seems to have made the response patterns relatively resistant to direct extinction. Direct extinction possibly would have been more successful had the extinction procedure involved a greater number of extinction trials, and future studies may benefit by including such procedures. Evaluating the stability of extinction over time and the possible resurgence of avoidance responding may also be beneficial. These issues notwithstanding, the current findings appear to provide an exciting empirical starting point for an investigation into novel extinction procedures based on the concept of derived relational responding.

\section{References}

AUGUSTON, E. M., \& DOUGHER, M. J. (1997). The transfer of avoidance evoking functions through stimulus equivalence classes. Journal of Behavioral Therapy and Experimental Psychiatry, 28, 181-191.

BARLOW, D. H. (2002). Anxiety and its disorders: The nature and treatment of anxiety and panic (2nd ed.). New York: Guilford Press. 
BARLOW, D. H., ALLEN, L. B., \& CHOATE, M. L. (2004). Toward a unified treatment for emotional disorders. Behavior Therapy, 35, 205-230.

BARNES, D., \& KEENAN, M. (1993). A transfer of functions through derived arbitrary and nonarbitrary stimulus relations. Journal of the Experimental Analysis of Behavior, 59, 61-81.

BARNES, D., \& ROCHE, B. (1996). Relational frame theory and stimulus equivalence are fundamentally different: A reply to Saunders. The Psychological Record, 46, 489-508.

BARNES, D., \& ROCHE, B. (1997). Relational frame theory and the experimental analysis of human sexuality. Applied and Preventive Psychology, 6, 117-135.

BARNES-HOLMES, D., \& BARNES-HOLMES, Y. (2000). Explaining complex behavior: Two perspectives on the concept of generalized operant classes. The Psychological Record, 50, 251-265.

COHEN, J. D., MACWHINNY, B., FLATT, M., \& PROVOST, J. (1993). PsyScope: A new graphic interactive environment for designing psychology experiments. Behavioral Research Methods, Instruments, and Computers, $25,257-271$.

DE ROSE, J. C., MCLLVANE, W. J., \& DUBE, W. V. (1988). Stimulus class formation and functional equivalence in moderately retarded individuals' conditional discrimination. Behavioural Processes, 17, 167-175.

DOUGHER, M. J., AUGUSTON, E., MARKHAM, M.R., GREENWAY, D.E., \& WULFERT, E.W. (1994). The transfer of respondent eliciting and extinction functions through stimulus equivalence classes. Journal of the Experimental Analysis of Behavior, 62, 331-351.

DOUGHER, M. J., \& MARKAM, M. R. (1994). Stimulus equivalence, functional equivalence and the transfer of function. In S. C. Hayes, L. J. Hayes, M. Sato, \& K. Ono (Eds.), Behavior Analysis of Language and Cognition (pp 71-90). Reno, NV: Context Press.

DYMOND, S., \& BARNES, D. (1995). A transfer of self-discrimination response functions in accordance with the arbitrarily applicable relations of sameness, more than, and less than. Journal of the Experimental Analysis of Behavior, 64, 163-184.

DYMOND, S., \& REHFELDT, R. (2000). Understanding complex behavior: The transformation of stimulus functions. The Behavior Analyst, 23, 239-254.

DYMOND, S., ROCHE, B., FORSYTH, J.P., WHELAN, R., \& RHODEN, J. (2007). Transformation of avoidance response functions in accordance with the relational frames of same and opposite. Journal of the Experimental Analysis of Behavior, 88, 249-262.

FORSYTH, J. P. (2000). A process-oriented behavioral approach to the etiology, maintenance, and treatment of anxiety-related disorders. In M. J. Dougher (Ed.), Clinical behavior analysis (pp. 153-180). Reno, NV: Context Press.

FORSYTH, J. P., \& EIFERT, G. H. (1996). Systemic alarms in fear conditioning I: A reappraisal of what is being conditioned. Behavior Therapy, 27, 441-462.

FRIMAN, P. C., HAYES, S. C., \& WILSON, K. G. (1994). Why behavior analysts should study emotion: The example of anxiety. Journal of Applied Behavior Analysis, 31, 137-156. 
GATCH, M. B., \& OSBORNE, J. G. (1989). Transfer of contextual stimulus function via equivalence class development. Journal of the Experimental Analysis of Behavior, 51, 369-378.

GREEN, G., SIGURDARDOTTIR, Z. G., \& SAUNDERS, R. R. (1991). The role of instructions in the transfer of ordinal functions through equivalence classes. Journal of the Experimental Analysis of Behavior, 55, 287-304.

HAYES, S. C., \& BARNES, D. (1997). Analyzing derived stimulus relations requires more than the concept of stimulus class. Journal of the Experimental Analysis of Behavior, 68, 235-270.

HAYES, S. C., BARNES-HOLMES, D., \& ROCHE, B. (2001). (Eds.). Relational Frame Theory: A Post-Skinnerian account of human language and cognition. New York: Plenum Press.

HAYES, S. C., KOHLENBERG, B. S., \& HAYES, L. J. (1991). The transfer of specific and general consequential functions through simple and conditional equivalence relations. Journal of the Experimental Analysis of Behavior, 56, 119-137.

HAYES, S. C., STROSAHL, K. D., \& WILSON, K. G. (1999). Acceptance and Commitment Therapy: An Experiential Approach to Behavior Change. New York: Guilford Press.

HAYES, S. C., WILSON, K. G., GIFFORD, E. V., FOLLETTE, V. M., \& STROSAHL, K. (1996). Experiential avoidance and behavioral disorders: A functional dimensional approach to diagnosis and treatment. Journal of Consulting and Clinical Psychology, 64, 1152-1168.

KOHLENBERG, B. S., HAYES, S. C., \& HAYES, L. J. (1991). The transfer of contextual control over equivalence classes: A possible model of social stereotyping. Journal of the Experimental Analysis of Behavior, 56, 505-518.

LANG, P. J., BRADLEY, M. M., \& CUTHBERT, B. N. (2001). International affective picture system (IAPS). Instruction manual and affective ratings. Technical Report A-5. Center for Research in Psychophysiology, University of Florida.

LAZAR, R. (1977). Extending sequence-class membership with matching to sample. Journal of the Experimental Analysis of Behavior, 27, 381-392.

MASUDA, A., HAYES, S. C., SACKETT, C. F., \& TWOHIG, M. P. (2004). Cognitive defusion and self-relevant negative thoughts: Examining the impact of a ninety year old technique. Behaviour Research and Therapy, 42, 477-485.

MOWER, O. H. (1960). Learning Theory and Behavior. New York: Wiley.

O'HORA, D., BARNES-HOLMES, D., ROCHE, B., \& SMEETS, P. M. (2004). Derived relational networks and control by novel instructions: A possible model of generative verbal responding. The Psychological Record, 54, 437-460.

O'HORA, D., ROCHE, B., BARNES-HOLMES, D., \& SMEETS, P. M. (2002). Response latencies to multiple derived stimulus relations: Testing two predictions of Relational Frame Theory. The Psychological Record, 52, 51-75.

OTTENBREIT, N. D., \& DOBSON, K. S. (2004). Avoidance and depression: The construction of the Cognitive-Behavioral Avoidance Scale. Behaviour Research and Therapy, 42, 293-313.

RACHMAN, S. J. (1977). The conditioning theory of fear acquisition: A critical examination. Behaviour Research and Therapy, 15, 375-387.

RACHMAN, S. (1991). Neo-conditioning and the classical theory of fear acquisition. Clinical Psychology Review, 11, 155-173. 
ROCHE, B., \& BARNES, D. (1996). Arbitrarily applicable relational responding and sexual categorization: A critical test of the derived difference relation. The Psychological Record, 46, 451-475.

ROCHE, B., \& BARNES, D. (1997). A transformation of respondently conditioned stimulus function in accordance with arbitrarily applicable relations. Journal of the Experimental Analysis of Behavior, 67, 275-301.

STEELE, D., \& HAYES, S. C. (1991). Stimulus equivalence and arbitrarily applicable relational responding. Journal of the Experimental Analysis of Behavior, 56, 519-555.

WOLPE, J. (1958). Psychotherapy by reciprocal inhibition. Oxford: Stanford University Press.

WHELAN, R., \& BARNES-HOLMES, D. (2004). The transformation of consequential functions in accordance with the relational frames of same and opposite. Journal of the Experimental Analysis of Behavior, 82, 177-195.

WHELAN, R., BARNES-HOLMES, D., \& DYMOND, S. (2006). The transformation of consequential functions in accordance with the relational frames of more-than and less-than. Journal of the Experimental Analysis of Behavior, 86, 317-335.

WULFERT, E., \& HAYES, S. C. (1988). Transfer of a conditional ordering response through conditional equivalence classes, Journal of the Experimental Analysis of Behavior, 50, 125-144. 